\title{
Electrodynamics in Uniformly Rotating Frames the Central Observer Point of View
}

\author{
A. Sfarti ${ }^{1}$ \\ ${ }^{1} \mathrm{CS}$ Dept, 387 Soda Hall, UC Berkeley \\ Email: egas@pacbell.net
}

\begin{abstract}
In the current paper we present a generalization of the transforms of the electromagnetic field from the frame co-moving with a rotating observer aligned with the axis of rotation into an inertial frame of reference. The solution is of great interest for real time applications, because earthbound laboratories are inertial only in approximation. We conclude by deriving the general form of the relativistic Doppler effect and of the relativistic aberration formulas for the case of uniformly rotating frames.
\end{abstract}

Keywords: Uniform rotation motion, general coordinate transformations, accelerated particles, planar electromagnetic waves, relativistic Doppler effect, relativistic aberration

PACS: 03.30.+p, 52.20.Dq, 52.70.Nc

\section{Introduction}

Real life applications include accelerating and rotating frames more often than the idealized case of inertial frames. Our daily experiments happen in the laboratories attached to the rotating, continuously accelerating Earth. Many books and papers have been dedicated to transformations between particular cases of rectilinear acceleration and/or rotation [1] and to the applications of such formulas [2-12]. The main idea of this paper is to generate a standard blueprint for a general solution that gives equivalent of the Lorentz transforms for the case of the transforms between an inertial frame and a uniformly rotating frame.

\section{Uniformly Rotating Motion - the Transforms of the Electromagnetic Field}

According to Moller [1], the transformation between the rotating frame $S^{\prime}$ attached to a uniformly rotating observer located along the rotation axis and an inertial, non-rotating frame $S$ attached to the center of rotation is:

$$
\left(\begin{array}{l}
x \\
y \\
z \\
t
\end{array}\right)=\text { Phy_rotation }\left(\begin{array}{l}
x^{\prime} \\
y^{\prime} \\
z^{\prime} \\
t^{\prime}
\end{array}\right)
$$

where:

$$
\text { Phy_rotation }=\left[\begin{array}{cccc}
\cos \Omega t & \sin \Omega t & 0 & 0 \\
-\sin \Omega t & \cos \Omega t & 0 & 0 \\
0 & 0 & 1 & 0 \\
0 & 0 & 0 & 1
\end{array}\right]
$$

In order to simplify things, we will use the notation: 
We also need the following:

$$
\text { Phy_rotation }=\left[\begin{array}{cccc}
a_{11} & a_{12} & 0 & 0 \\
a_{21} & a_{22} & 0 & 0 \\
0 & 0 & 1 & 0 \\
0 & 0 & 0 & 1
\end{array}\right]
$$

$$
\begin{aligned}
& \frac{\partial}{\partial x^{\prime}}=\frac{\partial}{\partial x} \frac{\partial x}{\partial x^{\prime}}+\frac{\partial}{\partial y} \frac{\partial y}{\partial x^{\prime}} \\
& \frac{\partial}{\partial y^{\prime}}=\frac{\partial}{\partial x} \frac{\partial x}{\partial y^{\prime}}+\frac{\partial}{\partial y} \frac{\partial y}{\partial y^{\prime}} \\
& \frac{\partial}{\partial z^{\prime}}=\frac{\partial}{\partial z} \\
& \frac{\partial}{\partial t^{\prime}}=\frac{\partial}{\partial t} \\
& {\left[\begin{array}{llll}
\frac{\partial x}{\partial x^{\prime}} & \frac{\partial x}{\partial y^{\prime}} & \frac{\partial x}{\partial z^{\prime}} & \frac{\partial x}{\partial t^{\prime}} \\
\frac{\partial y}{\partial x^{\prime}} & \frac{\partial y}{\partial y^{\prime}} & \frac{\partial y}{\partial z^{\prime}} & \frac{\partial y}{\partial t^{\prime}} \\
\frac{\partial z}{\partial x^{\prime}} & \frac{\partial z}{\partial y^{\prime}} & \frac{\partial z}{\partial z^{\prime}} & \frac{\partial z}{\partial t^{\prime}} \\
\frac{\partial t}{\partial x^{\prime}} & \frac{\partial t}{\partial y^{\prime}} & \frac{\partial t}{\partial z^{\prime}} & \frac{\partial t}{\partial t^{\prime}}
\end{array}\right]=\left[\begin{array}{cccc}
a_{11} & a_{12} & 0 & 0 \\
a_{21} & a_{22} & 0 & 0 \\
0 & 0 & 1 & 0 \\
0 & 0 & 0 & 1
\end{array}\right]}
\end{aligned}
$$

We also need the inverse transformation, from frame $S^{\prime}(\tau)$ into frame $S$ because it gives us:

$$
\left[\begin{array}{llll}
\frac{\partial x^{\prime}}{\partial x} & \frac{\partial x^{\prime}}{\partial y} & \frac{\partial x^{\prime}}{\partial z} & \frac{\partial x^{\prime}}{\partial t} \\
\frac{\partial y^{\prime}}{\partial x} & \frac{\partial y^{\prime}}{\partial y} & \frac{\partial y^{\prime}}{\partial z} & \frac{\partial y^{\prime}}{\partial t} \\
\frac{\partial z^{\prime}}{\partial x} & \frac{\partial z^{\prime}}{\partial y} & \frac{\partial z^{\prime}}{\partial z} & \frac{\partial z^{\prime}}{\partial t} \\
\frac{\partial t^{\prime}}{\partial x} & \frac{\partial t^{\prime}}{\partial y} & \frac{\partial t^{\prime}}{\partial z} & \frac{\partial t^{\prime}}{\partial t}
\end{array}\right]=\left[\begin{array}{cccc}
b_{11} & b_{12} & 0 & 0 \\
b_{21} & b_{22} & 0 & 0 \\
0 & 0 & 1 & 0 \\
0 & 0 & 0 & 1
\end{array}\right]=\left[\begin{array}{cccc}
a_{11} & -a_{12} & 0 & 0 \\
-a_{21} & a_{22} & 0 & 0 \\
0 & 0 & 1 & 0 \\
0 & 0 & 0 & 1
\end{array}\right]
$$

The electromagnetic potential, by virtue of being a 4 -vector transforms the same way as described by (2.1):

$$
\left(\begin{array}{c}
A_{x} \\
A_{y} \\
A_{z} \\
\varphi
\end{array}\right)=\text { Phy_rotation }\left(\begin{array}{c}
A_{x}^{\prime} \\
A_{y}^{\prime} \\
A_{z}^{\prime} \\
\varphi^{\prime}
\end{array}\right)
$$

In the inertial frame, the differential Maxwell equations in vacuum, in the absence of electric charge, are [1]:

$$
\begin{aligned}
& -E_{x}=\frac{\partial A_{x}}{\partial t}+c^{2} \frac{\partial \varphi}{\partial x} \\
& -E_{y}=\frac{\partial A_{y}}{\partial t}+c^{2} \frac{\partial \varphi}{\partial y} \\
& -E_{z}=\frac{\partial A_{z}}{\partial t}+c^{2} \frac{\partial \varphi}{\partial z}
\end{aligned}
$$


Let's start with (2.8):

$$
\mathbf{B}=\operatorname{curl} \mathbf{A}
$$

$$
\begin{gathered}
A_{x}=a_{11} A_{x}^{\prime}+a_{12} A_{y}^{\prime} \\
\frac{\partial A_{x}}{\partial t}=a_{11} \frac{\partial A_{x}^{\prime}}{\partial t^{\prime}}+a_{12} \frac{\partial A_{y}^{\prime}}{\partial t^{\prime}} \\
\frac{\partial \varphi}{\partial x}=\frac{\partial \varphi}{\partial x^{\prime}} \frac{\partial x^{\prime}}{\partial x}+\frac{\partial \varphi}{\partial y^{\prime}} \frac{\partial y^{\prime}}{\partial x}+\frac{\partial \varphi}{\partial z^{\prime}} \frac{\partial z^{\prime}}{\partial x}=a_{11} \frac{\partial \varphi^{\prime}}{\partial x^{\prime}}-a_{21} \frac{\partial \varphi^{\prime}}{\partial y^{\prime}}=a_{11} \frac{\partial \varphi^{\prime}}{\partial x^{\prime}}+a_{12} \frac{\partial \varphi^{\prime}}{\partial y^{\prime}} \\
-E_{x}=\frac{\partial A_{x}}{\partial t}+c^{2} \frac{\partial \varphi}{\partial x}=a_{11} \frac{\partial A_{x}^{\prime}}{\partial t^{\prime}}+a_{12} \frac{\partial A_{y}^{\prime}}{\partial t^{\prime}}+c^{2} a_{11} \frac{\partial \varphi^{\prime}}{\partial x^{\prime}}+c^{2} a_{12} \frac{\partial \varphi^{\prime}}{\partial y^{\prime}}=-a_{11} E_{x}^{\prime}-a_{12} E_{y}^{\prime}
\end{gathered}
$$

So:

$$
E_{x}=a_{11} E_{x}^{\prime}+a_{12} E_{y}^{\prime}
$$

Moving on to the second Maxwell equation, (2.9):

$$
\begin{aligned}
\frac{\partial A_{y}}{\partial t} & =a_{21} \frac{\partial A_{x}^{\prime}}{\partial t^{\prime}}+a_{22} \frac{\partial A_{y}^{\prime}}{\partial t^{\prime}} \\
\frac{\partial \varphi}{\partial y}=a_{44} b_{12} \frac{\partial \varphi^{\prime}}{\partial x^{\prime}}+a_{44} b_{22} \frac{\partial \varphi^{\prime}}{\partial y^{\prime}} & =-a_{12} \frac{\partial \varphi^{\prime}}{\partial x^{\prime}}+a_{22} \frac{\partial \varphi^{\prime}}{\partial y^{\prime}}=a_{21} \frac{\partial \varphi^{\prime}}{\partial x^{\prime}}+a_{22} \frac{\partial \varphi^{\prime}}{\partial y^{\prime}} \\
E_{y} & =a_{21} E_{x}^{\prime}+a_{22} E_{y}^{\prime}
\end{aligned}
$$

Moving on to (2.10):

$$
\begin{gathered}
\frac{\partial A_{z}}{\partial t}=\frac{\partial A_{z}^{\prime}}{\partial t}=\frac{\partial A_{z}^{\prime}}{\partial t^{\prime}} \\
\frac{\partial \varphi}{\partial z}=\frac{\partial \varphi}{\partial z^{\prime}}=\frac{\partial \varphi^{\prime}}{\partial z^{\prime}} \\
-E_{z}=\frac{\partial A_{z}}{\partial t}+c^{2} \frac{\partial \varphi}{\partial z}=\frac{\partial A_{z}^{\prime}}{\partial t^{\prime}}+c^{2} \frac{\partial \varphi^{\prime}}{\partial z^{\prime}}=-E_{z}^{\prime}
\end{gathered}
$$

Therefore:

$$
\begin{gathered}
E_{z}=E_{z}^{\prime} \\
B_{z}=\frac{\partial A_{y}}{\partial x}-\frac{\partial A_{x}}{\partial y} \\
\frac{\partial A_{y}}{\partial x}=\frac{\partial}{\partial x}\left(A_{x}^{\prime} a_{21}+A_{y}^{\prime} a_{22}\right) \\
\frac{\partial A_{x}^{\prime}}{\partial x}=\frac{\partial A_{x}^{\prime}}{\partial x^{\prime}} b_{11}+\frac{\partial A_{x}^{\prime}}{\partial y^{\prime}} b_{21} \\
\frac{\partial A_{y}^{\prime}}{\partial x}=\frac{\partial A_{y}^{\prime}}{\partial x^{\prime}} b_{11}+\frac{\partial A_{y}^{\prime}}{\partial y^{\prime}} b_{21} \\
a_{21}\left(\frac{\partial A_{x}^{\prime}}{\partial x^{\prime}} b_{11}+\frac{\partial A_{x}^{\prime}}{\partial y^{\prime}} b_{21}\right)+a_{22}\left(\frac{\partial A_{y}^{\prime}}{\partial x^{\prime}} b_{11}+\frac{\partial A_{y}^{\prime}}{\partial y^{\prime}} b_{21}\right) \\
\frac{\partial A_{x}}{\partial y}=\frac{\partial}{\partial y}\left(A_{x}^{\prime} a_{11}+A_{y}^{\prime} a_{12}\right) \\
\frac{\partial A_{x}^{\prime}}{\partial y}=\frac{\partial A_{x}^{\prime}}{\partial x^{\prime}} b_{12}+\frac{\partial A_{x}^{\prime}}{\partial y^{\prime}} b_{22}
\end{gathered}
$$




$$
\begin{aligned}
& \frac{\partial A_{y}^{\prime}}{\partial y}=\frac{\partial A_{y}^{\prime}}{\partial x^{\prime}} b_{12}+\frac{\partial A_{y}^{\prime}}{\partial y^{\prime}} b_{22}+\frac{\partial A_{y}^{\prime}}{\partial t^{\prime}} b_{42} \\
& \frac{\partial A_{x}}{\partial y}=a_{11}\left(\frac{\partial A_{x}^{\prime}}{\partial x^{\prime}} b_{12}+\frac{\partial A_{x}^{\prime}}{\partial y^{\prime}} b_{22}\right)+a_{12}\left(\frac{\partial A_{y}^{\prime}}{\partial x^{\prime}} b_{12}+\frac{\partial A_{y}^{\prime}}{\partial y^{\prime}} b_{22}\right) \\
& B_{z}=\frac{\partial A_{y}}{\partial x}-\frac{\partial A_{x}}{\partial y}=\left(a_{21} b_{11}-a_{11} b_{12}\right) \frac{\partial A_{x}^{\prime}}{\partial x^{\prime}}+\left(a_{22} b_{21}-a_{12} b_{22}\right) \frac{\partial A_{y}^{\prime}}{\partial y^{\prime}}+ \\
& +\left(a_{21} b_{21} \frac{\partial A_{x}^{\prime}}{\partial y^{\prime}}-a_{12} b_{12} \frac{\partial A_{y}^{\prime}}{\partial x^{\prime}}\right)-\left(a_{11} b_{22} \frac{\partial A_{x}^{\prime}}{\partial y^{\prime}}-a_{22} b_{11} \frac{\partial A_{y}^{\prime}}{\partial x^{\prime}}\right)= \\
& =\left(a_{21} b_{21} \frac{\partial A_{x}^{\prime}}{\partial y^{\prime}}-a_{12} b_{12} \frac{\partial A_{y}^{\prime}}{\partial x^{\prime}}\right)-\left(a_{11} b_{22} \frac{\partial A_{x}^{\prime}}{\partial y^{\prime}}-a_{22} b_{11} \frac{\partial A_{y}^{\prime}}{\partial x^{\prime}}\right)=\left(a_{21}^{2}+a_{11}^{2}\right)\left(\frac{\partial A_{y}^{\prime}}{\partial x^{\prime}}-\frac{\partial A_{x}^{\prime}}{\partial y^{\prime}}\right)=B_{z}^{\prime} \\
& B_{z}=B_{z}^{\prime} \\
& B_{x}=\frac{\partial A_{z}}{\partial y}-\frac{\partial A_{y}}{\partial z} \\
& \frac{\partial A_{z}}{\partial y}=\frac{\partial A_{z}^{\prime}}{\partial y}=\frac{\partial A_{z}^{\prime}}{\partial x^{\prime}} \frac{\partial x^{\prime}}{\partial y}+\frac{\partial A_{z}^{\prime}}{\partial y^{\prime}} \frac{\partial y^{\prime}}{\partial y}+\frac{\partial A_{z}^{\prime}}{\partial t^{\prime}} \frac{\partial t^{\prime}}{\partial y}=\frac{\partial A_{z}^{\prime}}{\partial x^{\prime}} b_{12}+\frac{\partial A_{z}^{\prime}}{\partial y^{\prime}} b_{22} \\
& \frac{\partial A_{y}}{\partial z}=\frac{\partial A_{y}}{\partial z^{\prime}}=\frac{\partial}{\partial z^{\prime}}\left(A_{x}^{\prime} a_{21}+A_{z}^{\prime} a_{22}+\varphi^{\prime} a_{24}\right)=\frac{\partial A_{x}^{\prime}}{\partial z^{\prime}} a_{21}+\frac{\partial A_{y}^{\prime}}{\partial z^{\prime}} a_{22} \\
& B_{x}=\frac{\partial A_{z}^{\prime}}{\partial x^{\prime}} b_{12}+\frac{\partial A_{z}^{\prime}}{\partial y^{\prime}} b_{22}-\frac{\partial A_{x}^{\prime}}{\partial z^{\prime}} a_{21}-\frac{\partial A_{y}^{\prime}}{\partial z^{\prime}} a_{22}= \\
& =\left(\frac{\partial A_{z}^{\prime}}{\partial y^{\prime}} b_{22}-\frac{\partial A_{y}^{\prime}}{\partial z^{\prime}} a_{22}\right)+\left(\frac{\partial A_{z}^{\prime}}{\partial x^{\prime}} b_{12}-\frac{\partial A_{x}^{\prime}}{\partial z^{\prime}} a_{21}\right)=a_{22}\left(\frac{\partial A_{z}^{\prime}}{\partial y^{\prime}}-\frac{\partial A_{y}^{\prime}}{\partial z^{\prime}}\right)+a_{12}\left(\frac{\partial A_{x}^{\prime}}{\partial z^{\prime}}-\frac{\partial A_{z}^{\prime}}{\partial x^{\prime}}\right)= \\
& =a_{22} B_{x}^{\prime}+a_{12} B_{y}^{\prime} \\
& B_{x}=a_{22} B_{x}^{\prime}+a_{12} B_{y}^{\prime} \\
& B_{y}=\frac{\partial A_{x}}{\partial z}-\frac{\partial A_{z}}{\partial x} \\
& \frac{\partial A_{x}}{\partial z}=\frac{\partial A_{x}}{\partial z^{\prime}}=\frac{\partial}{\partial z^{\prime}}\left(A_{x}^{\prime} a_{11}+A_{z}^{\prime} a_{12}\right)=\frac{\partial A_{x}^{\prime}}{\partial z^{\prime}} a_{11}+\frac{\partial A_{y}^{\prime}}{\partial z^{\prime}} a_{12} \\
& \frac{\partial A_{z}}{\partial x}=\frac{\partial A_{z}^{\prime}}{\partial x}=\frac{\partial A_{z}^{\prime}}{\partial x^{\prime}} \frac{\partial x^{\prime}}{\partial x}+\frac{\partial A_{z}^{\prime}}{\partial y^{\prime}} \frac{\partial y^{\prime}}{\partial x}+\frac{\partial A_{z}^{\prime}}{\partial t^{\prime}} \frac{\partial t^{\prime}}{\partial x}=\frac{\partial A_{z}^{\prime}}{\partial x^{\prime}} b_{11}+\frac{\partial A_{z}^{\prime}}{\partial y^{\prime}} b_{21} \\
& \frac{\partial A_{x}}{\partial z}-\frac{\partial A_{z}}{\partial x}=\left(\frac{\partial A_{x}^{\prime}}{\partial z^{\prime}} a_{11}-\frac{\partial A_{z}^{\prime}}{\partial x^{\prime}} b_{11}\right)+\left(\frac{\partial A_{y}^{\prime}}{\partial z^{\prime}} a_{12}-\frac{\partial A_{z}^{\prime}}{\partial y^{\prime}} b_{21}\right)= \\
& =a_{11}\left(\frac{\partial A_{x}^{\prime}}{\partial z^{\prime}}-\frac{\partial A_{z}^{\prime}}{\partial x^{\prime}}\right)+a_{12}\left(\frac{\partial A_{y}^{\prime}}{\partial z^{\prime}}-\frac{\partial A_{z}^{\prime}}{\partial y^{\prime}}\right)=a_{11} B_{y}^{\prime}-a_{12} B_{x}^{\prime} \\
& B_{y}=a_{11} B_{y}^{\prime}-a_{12} B_{x}^{\prime}
\end{aligned}
$$

To summarize: 


$$
\begin{aligned}
& E_{x}=a_{11} E_{x}^{\prime}+a_{12} E_{y}^{\prime} \\
& B_{x}=a_{22} B_{x}^{\prime}+a_{12} B_{y}^{\prime}=a_{11} B_{x}^{\prime}+a_{12} B_{y}^{\prime} \\
& E_{y}=a_{21} E_{x}^{\prime}+a_{22} E_{y}^{\prime}=a_{11} E_{y}^{\prime}-a_{12} E_{x}^{\prime} \\
& B_{y}=a_{12} B_{x}^{\prime}+a_{11} B_{y}^{\prime}=a_{11} B_{y}^{\prime}-a_{12} B_{x}^{\prime} \\
& E_{z}=E_{z}^{\prime} \\
& B_{z}=B_{z}^{\prime}
\end{aligned}
$$

An immediate consequence is:

$$
\begin{aligned}
& E=E^{\prime} \\
& B=B^{\prime}
\end{aligned}
$$

Obviously, the quantity $E^{2}-(B c)^{2}$ is invariant with respect to the transforms (2.1). Other invariants will be studied in the next section.

Recasting (2.44) in matrix form allows us to notice another important property:

$$
\begin{aligned}
& \left(\begin{array}{l}
E_{x} \\
E_{y} \\
E_{z} \\
B_{z}
\end{array}\right)=\text { Phy_rotation }\left(\begin{array}{l}
E_{x}^{\prime} \\
E_{y}^{\prime} \\
E_{z}^{\prime} \\
B_{z}^{\prime}
\end{array}\right) \\
& \left(\begin{array}{l}
B_{x} \\
B_{y} \\
E_{z} \\
B_{z}
\end{array}\right)=\text { Phy_rotation }\left(\begin{array}{l}
B_{x}^{\prime} \\
B_{y}^{\prime} \\
E_{z}^{\prime} \\
B_{z}^{\prime}
\end{array}\right)
\end{aligned}
$$

The above produces the inverse transforms:

$$
\begin{aligned}
& \left(\begin{array}{l}
E_{x}^{\prime} \\
E_{y}^{\prime} \\
E_{z}^{\prime} \\
B_{z}^{\prime}
\end{array}\right)=\text { Phy_rotation }^{-1}\left(\begin{array}{l}
E_{x} \\
E_{y} \\
E_{z} \\
B_{z}
\end{array}\right) \\
& \left(\begin{array}{l}
B_{x}^{\prime} \\
B_{y}^{\prime} \\
E_{z}^{\prime} \\
B_{z}^{\prime}
\end{array}\right)=\text { Phy_rotation }{ }^{-1}\left(\begin{array}{l}
B_{x} \\
B_{y} \\
E_{z} \\
B_{z}
\end{array}\right)
\end{aligned}
$$

\subsection{Consequences}

\subsubsection{Maxwell laws in a uniformly rotating frame}

From the calculations in the previous section we deduce immediately that:

Indeed:

$$
\begin{aligned}
& \mathbf{E}^{\prime}=-\frac{\partial \mathbf{A}^{\prime}}{\partial t^{\prime}}-c^{2} \nabla \varphi^{\prime} \\
& \mathbf{B}^{\prime}=\operatorname{curl} \mathbf{A}^{\prime}
\end{aligned}
$$

$$
\begin{aligned}
& \frac{\partial \mathbf{A}^{\prime}}{\partial t^{\prime}}=\frac{\partial}{\partial t}\left(\mathbf{i} A_{x}^{\prime}+\mathbf{j} A_{y}^{\prime}+\mathbf{k} A_{z}^{\prime}\right)=\mathbf{i} \frac{\partial}{\partial t}\left(b_{11} A_{x}+b_{12} A_{y}\right)+\mathbf{j} \frac{\partial}{\partial t}\left(b_{21} A_{x}+b_{22} A_{y}\right)+\mathbf{k} \frac{\partial A_{z}}{\partial t}= \\
& =\mathbf{i}\left(b_{11} \frac{\partial A_{x}}{\partial t}+b_{12} \frac{\partial A_{y}}{\partial t}\right)+\mathbf{j}\left(b_{21} \frac{\partial A_{x}}{\partial t}+b_{22} \frac{\partial A_{y}}{\partial t}\right)+\mathbf{k} \frac{\partial A_{z}}{\partial t}
\end{aligned}
$$




$$
\begin{aligned}
& \nabla \varphi^{\prime}=\mathbf{i} \frac{\partial \varphi^{\prime}}{\partial x^{\prime}}+\mathbf{j} \frac{\partial \varphi^{\prime}}{\partial y^{\prime}}+\mathbf{k} \frac{\partial \varphi^{\prime}}{\partial z^{\prime}}=\mathbf{i}\left(\frac{\partial \varphi}{\partial x} \frac{\partial x}{\partial x^{\prime}}+\frac{\partial \varphi}{\partial y} \frac{\partial y}{\partial x^{\prime}}+\frac{\partial \varphi}{\partial z} \frac{\partial z}{\partial x^{\prime}}\right)+ \\
& +\mathbf{j}\left(\frac{\partial \varphi}{\partial x} \frac{\partial x}{\partial y^{\prime}}+\frac{\partial \varphi}{\partial y} \frac{\partial y}{\partial y^{\prime}}+\frac{\partial \varphi}{\partial z} \frac{\partial z}{\partial y^{\prime}}\right)+\mathbf{k} \frac{\partial \varphi}{\partial z}= \\
& =\mathbf{i}\left(a_{11} \frac{\partial \varphi}{\partial x}+a_{21} \frac{\partial \varphi}{\partial y}\right)+\mathbf{j}\left(a_{12} \frac{\partial \varphi}{\partial x}+a_{22} \frac{\partial \varphi}{\partial y}\right)+\mathbf{k} \frac{\partial \varphi}{\partial z} \\
& \frac{\partial \mathbf{A}^{\prime}}{\partial t^{\prime}}+c^{2} \nabla \varphi^{\prime}=\mathbf{i}\left(b_{11} \frac{\partial A_{x}}{\partial t}+b_{12} \frac{\partial A_{y}}{\partial t}\right)+\mathbf{j}\left(b_{21} \frac{\partial A_{x}}{\partial t}+b_{22} \frac{\partial A_{y}}{\partial t}\right)+\mathbf{k} \frac{\partial A_{z}}{\partial t}+ \\
& +\mathbf{i} c^{2}\left(a_{11} \frac{\partial \varphi}{\partial x}+a_{21} \frac{\partial \varphi}{\partial y}\right)+\mathbf{j} c^{2}\left(a_{12} \frac{\partial \varphi}{\partial x}+a_{22} \frac{\partial \varphi}{\partial y}\right)+\mathbf{k} c^{2} \frac{\partial \varphi}{\partial z}= \\
& =\mathbf{i}\left(b_{11} \frac{\partial A_{x}}{\partial t}+b_{12} \frac{\partial A_{y}}{\partial t}+a_{11} c^{2} \frac{\partial \varphi}{\partial x}+a_{21} c^{2} \frac{\partial \varphi}{\partial y}\right)+ \\
& +\mathbf{j}\left(b_{21} \frac{\partial A_{x}}{\partial t}+b_{22} \frac{\partial A_{y}}{\partial t}+c^{2} a_{12} \frac{\partial \varphi}{\partial x}+c^{2} a_{22} \frac{\partial \varphi}{\partial y}\right)+\mathbf{k}\left(\frac{\partial A_{z}}{\partial t}+c^{2} \frac{\partial \varphi}{\partial z}\right)= \\
& =\mathbf{i}\left(a_{11} E_{x}+a_{21} E_{y}\right)+\mathbf{j}\left(a_{12} E_{x}+a_{22} E_{y}\right)+\mathbf{k}\left(\frac{\partial A_{z}}{\partial t}+c^{2} \frac{\partial \varphi}{\partial z}\right)= \\
& =-\left(\mathbf{i} E_{x}^{\prime}+\mathbf{j} E_{y}^{\prime}+\mathbf{k} E_{z}^{\prime}\right)=-\mathbf{E}^{\prime} \\
& \operatorname{curl} \mathbf{A}^{\prime}=\left[\begin{array}{ccc}
\mathbf{i} & \mathbf{j} & \mathbf{k} \\
\frac{\partial}{\partial x^{\prime}} & \frac{\partial}{\partial y^{\prime}} & \frac{\partial}{\partial z^{\prime}} \\
A_{x}^{\prime} & A_{y}^{\prime} & A_{z}^{\prime}
\end{array}\right]=\mathbf{i}\left(\frac{\partial A_{z}^{\prime}}{\partial y^{\prime}}-\frac{\partial A_{y}^{\prime}}{\partial z^{\prime}}\right)+\mathbf{j}\left(\frac{\partial A_{x}^{\prime}}{\partial z^{\prime}}-\frac{\partial A_{z}^{\prime}}{\partial x^{\prime}}\right)+\mathbf{k}\left(\frac{\partial A_{y}^{\prime}}{\partial x^{\prime}}-\frac{\partial A_{x}^{\prime}}{\partial y^{\prime}}\right) \\
& \frac{\partial A_{z}^{\prime}}{\partial y^{\prime}}=\frac{\partial A_{z}}{\partial y} \\
& \frac{\partial A_{y}^{\prime}}{\partial z^{\prime}}=\frac{\partial A_{y}}{\partial z} \\
& \frac{\partial A_{x}^{\prime}}{\partial z^{\prime}}=\frac{\partial A_{x}^{\prime}}{\partial z}=\frac{\partial}{\partial z}\left(b_{11} A_{x}+b_{12} A_{y}\right)=b_{11} \frac{\partial A_{x}}{\partial z}+b_{12} \frac{\partial A_{y}}{\partial z}=a_{11} \frac{\partial A_{x}}{\partial z}-a_{12} \frac{\partial A_{y}}{\partial z} \\
& \frac{\partial A_{z}^{\prime}}{\partial x^{\prime}}=\frac{\partial A_{z}}{\partial x^{\prime}}=\frac{\partial A_{z}}{\partial x} \frac{\partial x}{\partial x^{\prime}}+\frac{\partial A_{z}}{\partial y} \frac{\partial y}{\partial x^{\prime}}+\frac{\partial A_{z}}{\partial z} \frac{\partial z}{\partial x^{\prime}}=a_{11} \frac{\partial A_{z}}{\partial x}+a_{21} \frac{\partial A_{z}}{\partial y}=a_{11} \frac{\partial A_{z}}{\partial x}-a_{12} \frac{\partial A_{z}}{\partial y} \\
& \frac{\partial A_{y}^{\prime}}{\partial x^{\prime}}=\frac{\partial A_{y}}{\partial x^{\prime}}=\frac{\partial A_{y}}{\partial x} \frac{\partial x}{\partial x^{\prime}}+\frac{\partial A_{y}}{\partial y} \frac{\partial y}{\partial x^{\prime}}+\frac{\partial A_{y}}{\partial z} \frac{\partial z}{\partial x^{\prime}}=a_{11} \frac{\partial A_{y}}{\partial x}+a_{21} \frac{\partial A_{y}}{\partial y}=a_{11} \frac{\partial A_{y}}{\partial x}-a_{12} \frac{\partial A_{y}}{\partial y} \\
& \frac{\partial A_{x}^{\prime}}{\partial y^{\prime}}=\frac{\partial A_{x}^{\prime}}{\partial y}=\frac{\partial}{\partial y}\left(b_{11} A_{x}+b_{12} A_{y}\right)=b_{11} \frac{\partial A_{x}}{\partial y}+b_{12} \frac{\partial A_{y}}{\partial y}=a_{11} \frac{\partial A_{x}}{\partial y}-a_{12} \frac{\partial A_{y}}{\partial y}
\end{aligned}
$$

Therefore:

$$
\begin{aligned}
& \operatorname{curl} \mathbf{A}^{\prime}=\mathbf{i}\left(\frac{\partial A_{z}}{\partial y}-\frac{\partial A_{y}}{\partial z}\right)+\mathbf{j}\left[a_{11}\left(\frac{\partial A_{x}}{\partial z}-\frac{\partial A_{z}}{\partial x}\right)+a_{12}\left(\frac{\partial A_{z}}{\partial y}-\frac{\partial A_{y}}{\partial z}\right)\right]+ \\
& +\mathbf{k}\left[a_{11}\left(\frac{\partial A_{y}}{\partial x} \frac{\partial A_{x}}{\partial y}\right)+a_{12}\left(\frac{\partial A_{y}}{\partial y}-\frac{\partial A_{y}}{\partial y}\right)\right]=\mathbf{i} B_{x}^{\prime}+\mathbf{j} B_{y}^{\prime}+\mathbf{k} B_{z}^{\prime}=\mathbf{B}^{\prime}
\end{aligned}
$$

2.2.2. The gauge invariance condition in a uniformly rotating frame

$$
\operatorname{div} \mathbf{A}^{\prime}+\frac{\partial \varphi^{\prime}}{\partial t^{\prime}}=\operatorname{div} \mathbf{A}+\frac{\partial \varphi}{\partial t}=0
$$




$$
\begin{gathered}
\operatorname{div} \mathbf{A}^{\prime}=\frac{\partial A_{x}^{\prime}}{\partial x^{\prime}}+\frac{\partial A_{y}^{\prime}}{\partial y^{\prime}}+\frac{\partial A_{z}^{\prime}}{\partial z^{\prime}}=\frac{\partial A_{x}^{\prime}}{\partial x^{\prime}}+\frac{\partial A_{y}^{\prime}}{\partial y^{\prime}}+\frac{\partial A_{z}}{\partial z} \\
\frac{\partial A_{x}^{\prime}}{\partial x^{\prime}}=b_{11}\left(\frac{\partial A_{x}}{\partial x} a_{11}+\frac{\partial A_{x}}{\partial y} a_{21}\right)+b_{12}\left(\frac{\partial A_{y}}{\partial x} a_{11}+\frac{\partial A_{y}}{\partial y} a_{21}\right)+b_{14}\left(\frac{\partial \varphi}{\partial x} a_{11}+\frac{\partial \varphi}{\partial y} a_{21}\right) \\
\frac{\partial A_{y}^{\prime}}{\partial y^{\prime}}=b_{21}\left(\frac{\partial A_{x}}{\partial x} a_{12}+\frac{\partial A_{x}}{\partial y} a_{22}\right)+b_{22}\left(\frac{\partial A_{y}}{\partial x} a_{12}+\frac{\partial A_{y}}{\partial y} a_{22}\right)+b_{24}\left(\frac{\partial \varphi}{\partial x} a_{12}+\frac{\partial \varphi}{\partial y} a_{22}\right) \\
\operatorname{div} \mathbf{A}^{\prime}+\frac{\partial \varphi^{\prime}}{\partial t^{\prime}}=\left(b_{11} a_{11}+b_{21} a_{12}\right) \frac{\partial A_{x}}{\partial x}+\left(b_{11} a_{21}+b_{21} a_{22}\right) \frac{\partial A_{x}}{\partial y}+\left(b_{12} a_{11}+b_{22} a_{12}\right) \frac{\partial A_{y}}{\partial x}+\left(\frac{\partial \varphi}{\partial x} a_{14}+\frac{\partial \varphi}{\partial y} a_{24}+\frac{\partial \varphi}{\partial t} a_{44}\right) \\
\left.+\left(b_{14} a_{11}+b_{22} a_{12} a_{22}\right) \frac{\partial A_{y}}{\partial y}+b_{44} a_{14}\right) \frac{\partial \varphi}{\partial x}+\left(b_{14} a_{21}+b_{24} a_{22}+b_{44} a_{24}\right) \frac{\partial \varphi}{\partial y}+a_{44} b_{44} \frac{\partial \varphi}{\partial t}+\frac{\partial A_{z}}{\partial z}=\frac{\partial A_{x}}{\partial x}+\frac{\partial A_{y}}{\partial y}+\frac{\partial A_{z}}{\partial z}+\frac{\partial \varphi}{\partial t}
\end{gathered}
$$

Equality (2.65) results into:

$$
\operatorname{div} \mathbf{A}^{\prime}+\frac{\partial \varphi^{\prime}}{\partial t^{\prime}}=\operatorname{div} \mathbf{A}+\frac{\partial \varphi}{\partial t}=0
$$

Equalities (2.48) and (2.66) result into Maxwell's equations having the same exact form in the uniformly rotating frame as the equations in the inertial frames with the immediate consequence that light speed in vacuum in a uniformly a rotating frame is "c". Indeed, (2.48) results into:

$$
\begin{aligned}
& \frac{1}{c^{2}} \frac{\partial^{2} \mathbf{E}^{\prime}}{\partial t^{\prime 2}}-\nabla^{2} \mathbf{E}^{\prime}=0 \\
& \frac{1}{c^{2}} \frac{\partial^{2} \mathbf{B}^{\prime}}{\partial t^{\prime 2}}-\nabla^{2} \mathbf{B}^{\prime}=0
\end{aligned}
$$

The above means that electromagnetic waves propagate in vacuum, in uniformly rotating frames, at the same speed as they propagate in inertial frames. In the next section we will derive this plus some other very interesting facts through a different approach.

\section{Planar Wave Transformation and Speed of Light in a Uniformly Rotating Frame}

In this section we apply the formalism derived in the previous paragraph in order to obtain the transform of a planar wave. Assume that a planar wave is propagating along the y' axis in the accelerated frame $S^{\prime}(\tau)$. The wave has the electric component $\mathbf{E}_{x}^{\prime}$ and the magnetic component $\mathbf{B}_{z}^{\prime}$ along the x' and z' axes, respectively. The components equations are (see fig.1):

$$
\begin{aligned}
& \mathbf{E}_{x}^{\prime}=E_{0 x}^{\prime} \cos \left(\Omega^{\prime} t^{\prime}-k_{y}^{\prime} y^{\prime}+\theta^{\prime}\right) \mathbf{e}_{x} \\
& \mathbf{B}_{z}^{\prime}=B_{0 z}^{\prime} \cos \left(\Omega^{\prime} t^{\prime}-k_{y}^{\prime} y^{\prime}+\theta^{\prime}\right) \mathbf{e}_{z}
\end{aligned}
$$

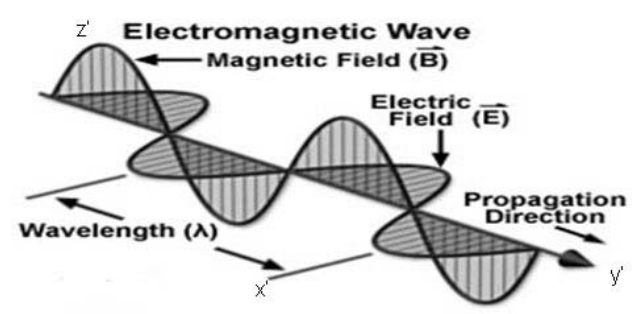

Figure 1. The electromagnetic wave

On the other hand: 


$$
\left(\begin{array}{l}
x^{\prime} \\
y^{\prime} \\
z^{\prime} \\
t^{\prime}
\end{array}\right)=\left[\begin{array}{cccc}
b_{11} & b_{12} & 0 & 0 \\
b_{21} & b_{22} & 0 & 0 \\
0 & 0 & 1 & 0 \\
0 & 0 & 0 & 1
\end{array}\right]\left(\begin{array}{l}
x \\
y \\
z \\
t
\end{array}\right)
$$

In frame $\mathrm{S}$, the wave equation is either:

$$
\begin{aligned}
& \left.E_{x}=(\gamma \cos \alpha \cos \beta+\sin \alpha \sin \beta) E_{x}^{\prime}-(u \gamma \cos \beta) B_{z}^{\prime}\right\}= \\
& =\left[(\gamma \cos \alpha \cos \beta+\sin \alpha \sin \beta) E_{0 x}^{\prime}-(u \gamma \cos \beta) B_{0 z}^{\prime}\right]^{*} \\
& * \cos \left[\left(\Omega^{\prime} b_{44}-k_{y}^{\prime} b_{24}\right) t-\left(k_{y}^{\prime} b_{22}-\Omega^{\prime} b_{42}\right) y-\left(k_{y}^{\prime} b_{21}-\Omega^{\prime} b_{41}\right) x+\theta^{\prime}\right] \\
& E_{y}=\left[(\gamma \cos \alpha \sin \beta-\sin \alpha \cos \beta) E_{0 x}^{\prime}-(u \gamma \sin \beta) B_{0 z}^{\prime}\right] * \\
& * \cos \left[\left(\Omega^{\prime} b_{44}-k_{y}^{\prime} b_{24}\right) t-\left(k_{y}^{\prime} b_{22}-\Omega^{\prime} b_{42}\right) y-\left(k_{y}^{\prime} b_{21}-\Omega^{\prime} b_{41}\right) x+\theta^{\prime}\right] \\
& B_{z}=\gamma\left(B_{0 z}^{\prime}-\frac{u \cos \alpha}{c^{2}} E_{0 x}^{\prime}\right)^{*} \\
& * \cos \left[\left(\Omega^{\prime} b_{44}-k_{y}^{\prime} b_{24}\right) t-\left(k_{y}^{\prime} b_{22}-\Omega^{\prime} b_{42}\right) y-\left(k_{y}^{\prime} b_{21}-\Omega^{\prime} b_{41}\right) x+\theta^{\prime}\right]
\end{aligned}
$$

or:

$$
\begin{aligned}
& \mathbf{E}_{x}=E_{0 x} \cos \left(\Omega t-k_{x} x-k_{y} y-k_{z} z+\theta\right) \mathbf{e}_{x} \\
& \mathbf{E}_{y}=E_{0 y} \cos \left(\Omega t-k_{x} x-k_{y} y-k_{z} z+\theta\right) \mathbf{e}_{y} \\
& \mathbf{B}_{z}=B_{0 z} \cos \left(\Omega t-k_{x} x-k_{y} y-k_{z} z+\theta\right) \mathbf{e}_{z}
\end{aligned}
$$

Comparing (3.4) and (3.3) we obtain:

$$
\begin{aligned}
& E_{0 x}=(\gamma \cos \alpha \cos \beta+\sin \alpha \sin \beta) E_{0 x}^{\prime}-(u \cos \beta) B_{0 z}^{\prime} \\
& \Omega=\Omega^{\prime} b_{44}-k_{y}^{\prime} b_{24} \\
& k_{x}=k_{y}^{\prime} b_{21}-\Omega^{\prime} b_{41} \\
& k_{y}=k_{y}^{\prime} b_{22}-\Omega^{\prime} b_{42} \\
& k_{z}=0 \\
& \theta=\theta^{\prime} \\
& c=\frac{\Omega}{\sqrt{k_{x}^{2}+k_{y}^{2}}}=\frac{\Omega^{\prime} b_{44}-k_{y}^{\prime} b_{24}}{\sqrt{\left(k_{y}^{\prime} b_{21}-\Omega^{\prime} b_{41}\right)^{2}+\left(k_{y}^{\prime} b_{22}-\Omega^{\prime} b_{42}\right)^{2}}} \\
& \frac{\Omega^{\prime 2}}{k^{\prime 2}}\left(c^{2} b_{41}^{2}+c^{2} b_{42}^{2}-b_{44}^{2}\right)-2 \frac{\Omega^{\prime}}{k^{\prime}}\left(c^{2} b_{21} b_{41}+c^{2} b_{22} b_{42}-b_{24} b_{44}\right)+c^{2} b_{21}^{2}+c^{2} b_{22}^{2}-b_{24}^{2}=0 \\
& c^{2}\left(b_{41}^{2}+b_{42}^{2}\right)-b_{44}^{2}=c^{2}\left(\left(\frac{u \gamma \sin \beta}{c^{2}}\right)^{2}+\left(\frac{u \gamma \cos \beta}{c^{2}}\right)^{2}\right)-\gamma^{2}=\frac{u^{2} \gamma^{2}}{c^{2}}-\gamma^{2}=-1 \\
& c^{2}\left(b_{21} b_{41}+b_{22} b_{42}\right)-b_{24} b_{44}=-u \gamma \sin \beta(\sin \alpha \cos \beta-\gamma \cos \alpha \sin \beta)+ \\
& +u \gamma \cos \beta(\sin \alpha \sin \beta+\gamma \cos \alpha \cos \beta)-u \gamma^{2} \cos \alpha=0 \\
& c^{2}\left(b_{21}^{2}+b_{22}^{2}\right)-b_{24}^{2}=c^{2}\left[(\sin \alpha \cos \beta-\gamma \cos \alpha \sin \beta)^{2}+(\sin \alpha \sin \beta+\gamma \cos \alpha \cos \beta)^{2}\right]- \\
& -(u \gamma \cos \alpha)^{2}=c^{2}\left(\sin ^{2} \alpha+\gamma^{2} \cos ^{2} \alpha\right)-u^{2} \gamma^{2} \cos ^{2} \alpha=c^{2} \\
& -\frac{\Omega^{\prime 2}}{k^{\prime 2}}+c^{2}=0 \\
& \frac{\Omega^{\prime}}{k^{\prime}}= \pm c
\end{aligned}
$$

We can now calculate the phase light speed in the rotating frame: 


$$
v_{p}^{\prime}=\frac{\Omega^{\prime}}{k^{\prime}}=\frac{\Omega}{k}= \pm c
$$

So, the light speed in the rotating frame equals the light speed in the inertial frame, $c$.

We can now proceed to calculating the amplitude and the phase transformation between the inertial and the rotating frame:

$$
\theta=\theta^{\prime}
$$

The general equation of the Doppler effect is:

$$
\begin{aligned}
& \Omega=\Omega^{\prime} b_{44}-k_{y}^{\prime} b_{24}=\Omega^{\prime}\left(b_{44}-\frac{b_{24}}{c}\right)=\Omega^{\prime}\left(\gamma-\frac{u \gamma \cos \alpha}{c}\right)=\gamma\left(1-\frac{u \cos \alpha}{c}\right) \Omega^{\prime} \\
& \gamma=\frac{1}{\sqrt{1-\frac{u^{2}}{c^{2}}}} \\
& u=r \omega \\
& \alpha=\omega \gamma \tau
\end{aligned}
$$

The general equations for aberration are:

$$
\begin{aligned}
& k_{x}=k_{y}^{\prime} b_{21}-\Omega^{\prime} b_{41}=k^{\prime} b_{21}-\Omega^{\prime} b_{41}=k^{\prime}\left(b_{21}-b_{41} c\right)=k^{\prime}\left(\sin \alpha \cos \beta-\gamma \cos \alpha \sin \beta-\frac{u \gamma \sin \beta}{c}\right) \\
& k_{y}=k_{y}^{\prime} b_{22}-\Omega^{\prime} b_{42}=k^{\prime} b_{22}-\Omega^{\prime} b_{42}=k^{\prime}\left(b_{22}-b_{42} c\right)=k^{\prime}\left(\sin \alpha \sin \beta+\gamma \cos \alpha \cos \beta+\frac{u \gamma \cos \beta}{c}\right) \\
& k_{z}=0 \\
& \beta=\omega \gamma^{2} \tau
\end{aligned}
$$

\section{General Case of Rotation about an Arbitrary Axis}

In a prior paper we have shown [12] that the particular transformation (2.1) can be generalized for the case of arbitrary direction. The general case is treated by transforming the problem into the particular case treated in [1] through a transformation into the "canonical case", followed by an application of the transformation from the rotating frame into the inertial frame, ending with the inverse of the first transformation, as shown below:

$$
\begin{aligned}
& \left(\begin{array}{l}
x \\
y \\
z \\
t
\end{array}\right)=\left(R r^{-1 * P h y \_r o t a t i o n} * R r\right)\left(\begin{array}{l}
x^{\prime} \\
y^{\prime} \\
z^{\prime} \\
t^{\prime}
\end{array}\right) \\
& R r=\operatorname{Rot}\left(\mathbf{e}_{x}\right)_{90^{0}} * \operatorname{Rot}\left(\mathbf{e}_{y}\right)_{90^{0}-\varphi} * \operatorname{Rot}_{y}
\end{aligned}
$$

$\operatorname{Rot}\left(\mathbf{e}_{y}\right)_{90^{0}-\varphi} * \operatorname{Rot}_{y}$ aligns $\boldsymbol{\omega}$ with $\mathbf{e}_{y}$. The second step is comprised by another rotation around the $\mathrm{x}-$ axis by $90^{\circ}$ that aligns $\boldsymbol{\omega}$ with $\mathbf{e}_{z}$ :

$$
\operatorname{Rot}\left(\mathbf{e}_{x}\right)_{90^{0}}=\left[\begin{array}{cccc}
1 & 0 & 0 & 0 \\
0 & 0 & -1 & 0 \\
0 & 1 & 0 & 0 \\
0 & 0 & 0 & 1
\end{array}\right]
$$




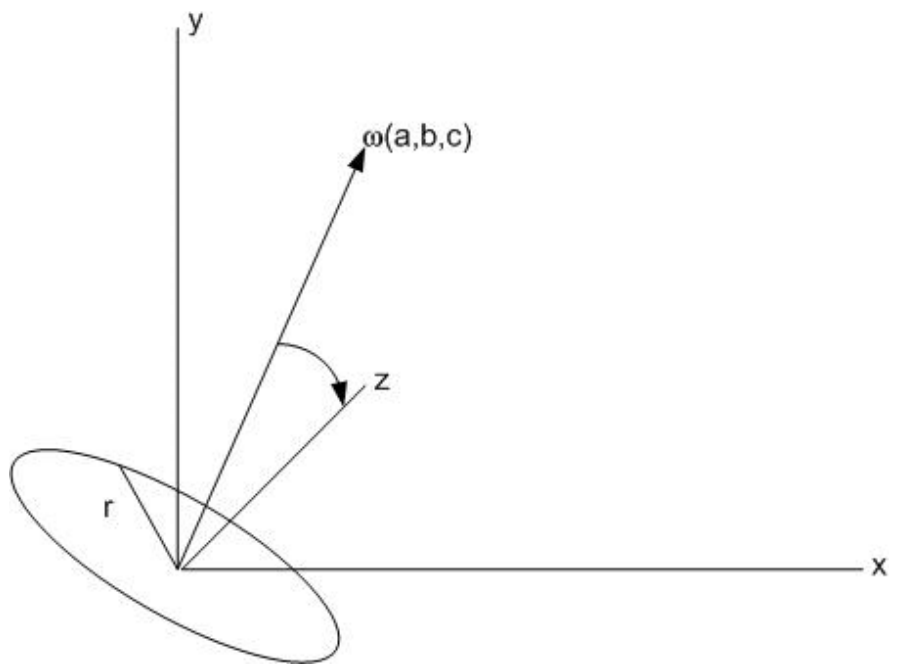

Figure 2. Uniform rotation with arbitrary direction of angular velocity

Expression (4.1) gives the solution for the general case, of arbitrary angular velocity direction. Rewriting (2.45) in tensor form we get:

$$
\left[\begin{array}{cccc}
E_{x} & B_{x} & * & * \\
E_{y} & * & B_{y} & * \\
* & E_{z} & * & * \\
* & * & * & B_{z}
\end{array}\right]=\left[\begin{array}{cccc}
\gamma \cos \alpha \cos \beta+\sin \alpha \sin \beta & \gamma \sin \alpha \cos \beta-\cos \alpha \sin \beta & -u \gamma \cos \beta & 0 \\
\gamma \cos \alpha \sin \beta-\sin \alpha \cos \beta & \gamma \sin \alpha \sin \beta+\cos \alpha \cos \beta & -u \gamma \sin \beta & 0 \\
u \gamma \cos \alpha & -u \gamma \sin \alpha & 0 & \gamma \\
0 & -\frac{u \gamma \cos \alpha}{c^{2}} & -\frac{u \gamma \sin \alpha}{c^{2}} & \gamma
\end{array}\right]\left[\begin{array}{cccc}
E_{x}^{\prime} & B_{x}^{\prime} & B_{y}^{\prime} & 0 \\
E_{y}^{\prime} & B_{y}^{\prime} & B_{x}^{\prime} & E_{x}^{\prime} \\
B_{z}^{\prime} & \frac{E_{z}^{\prime}}{c^{2}} & \frac{E_{z}^{\prime}}{c^{2}} & E_{y}^{\prime} \\
0 & E_{z}^{\prime} & 0 & B_{z}^{\prime}
\end{array}\right]
$$

The asterisks represent entries with no particular physical meaning, we do not care about them. Then, the general transform is:

$$
R r^{-1} *\left[\begin{array}{cccc}
\gamma \cos \alpha \cos \beta+\sin \alpha \sin \beta & \gamma \sin \alpha \cos \beta-\cos \alpha \sin \beta & -u \gamma \cos \beta & 0 \\
\gamma \cos \alpha \sin \beta-\sin \alpha \cos \beta & \gamma \sin \alpha \sin \beta+\cos \alpha \cos \beta & -u \sin \beta & 0 \\
u \gamma \cos \alpha & -u \gamma \sin \alpha & 0 & \gamma \\
0 & -\frac{u \gamma \cos \alpha}{c^{2}} & -\frac{u \gamma \sin \alpha}{c^{2}} & \gamma
\end{array}\right] * R r
$$

\section{Conclusions}

We constructed the general transforms from the uniformly rotating frame into an inertial frame of reference $S$. The solution is of great interest for real life applications, because our earth-bound laboratories are inertial only in approximation; in real life, the laboratories are accelerated and rotate. We produced a blueprint for generalizing the solutions for the arbitrary cases and we concluded with an application that explains the general case of planar electromagnetic waves. A very interesting consequence is the fact that light speed in vacuum in the rotating frames is "c". A second interesting consequence is that rotation induces aberration.

\section{Acknowledgements}

The author is grateful to the anonymous reviewer who reminded him of the equivalence between uniform gravitational fields and uniformly accelerated frames, including uniformly rotating ones [13].

\section{References}

1. Moller, C. "The Theory of Relativity," Oxford Press (1960). 
2. Thomas, L. H. "Motion of the spinning electron," Nature. 117 (1926).

3. Ben-Menahem, A. "Wigner's rotation revisited," Am. J. Phys. 53 (1985).

4. Ben-Menahem, S. "The Thomas precession and velocityspace curvature," J. Math. Phys. 27 (1986).

5. Kroemer, H. "The Thomas precession factor in spin-orbit interaction" Am J. Physics. 72 (2004).

6. Rhodes, J. A, "Semon, M. D. "Relativistic velocity space, Wigner rotation and Thomas precession," Am. J. Phys. 72, (2005).

7. Malykin, G. B. "Thomas precession: correct and incorrect solutions," Phys. Usp. 49 (8): (2006).

8. Krivoruchenko, M. I. "Rotation of the swing plane of Foucault's pendulum and Thomas spin preession: Two faces of one coin," Phys. Usp., 52. 8 (2009)

9. Sfarti, A. "Hyperbolic Motion Treatment for Bell's Spaceship Experiment," Fizika A, 18, 2 (2009)

10. Sfarti, A. "Coordinate Time Hyperbolic Motion for Bell's Spaceship Experiment," Fizika A, 19, 3 (2010)

11. Sfarti, A. "Relativity solution for "Twin paradox": a comprehensive solution," IJP, 86, 10 (2012)

12. Sfarti, A. "Generalization of Coordinate Transformations between Accelerated and Inertial Frames - General Formulas of Thomas Precession," JAPSI, 8, 2, (2017)

13. C. Corda, "The Mössbauer rotor experiment and the general theory of relativity," Ann. Phys. 368, 258 (2016). 